\title{
Revalidación psicométrica del cuestionario de actitudes hacia la matemática en
}

\section{estudiantes universitarios}

\author{
Farías Mata, Mariana Alejandra \\ Instituto Universitario de Tecnología "Dr. Federico Rivero Palacio". \\ Área de Desarrollo Humano. Caracas - Venezuela
}

La correspondencia relacionada con este artículo debe enviarse a: Mariana Alejandra Farías Mata. Carretera vía El Cují. Urbanización Altos de la Peña. Calle principal. Quinta M\&P. Municipio Los Salias. San Antonio de Los Altos. Estado Miranda. Zona postal 1204. Teléfonos: Habitación: 00582128926677 Celular: 00584127239351

Email:

Resumen. Este trabajo reporta los procedimientos de validación y confiabilidad obtenidos en la evaluación psicométrica del "Cuestionario de Actitudes hacia la Matemática" diseñado en 2007, como parte de una investigación sobre los nuevos enfoques en la enseñanza-aprendizaje de la matemática en estudiantes universitarios. A partir de sus resultados, se realizó la revalidación de las propiedades del "CAM". Para lograr este propósito, se aplicó este instrumento en el año 2013 a 404 estudiantes en una universidad tecnológica, cursantes de carreras que tienen diferentes niveles de contenido matemático o que ven asignaturas cuyo basamento es el conocimiento matemático. Finalmente, se analizan los resultados del proceso de revalidación y se sugieren algunas consideraciones sobre el enfoque, contribuciones y beneficios que podrían obtenerse con la aplicación de este cuestionario, para recopilar información sobre las actitudes en poblaciones de estudiantes universitarios.

Palabras clave: cuestionario, fiabilidad, validez, matemática, medición

\begin{abstract}
This job report the procedures of validation and reliability review, used in order to do the psychometrical evaluation of the "Questionnaire of Attitudes towards Mathematics" designed in 2007, as part of a research about new approaches in teaching - learning in university students. Starting from its results, we intend to re-validate the "QAM" properties. Whit this purpose we apply in 2013, this instrument to 404 students in a technological university, in careers which have different levels of mathematical contents or subjects that uses mathematics knowledge as basement. Finally we analyze the results of the re-validation process and we suggest some considerations regard to the scope and contributions that could be obtain whit the application of this questionnaire, in order to collect information about attitudes in university student's populations.
\end{abstract}

Keywords: questionnaire, reliability, validity, mathematics, measurement

\section{INTRODUCCIÓN}

Desde mediados del siglo XX, se han realizado esfuerzos en la investigación sobre actitudes en el campo de la educación matemática. Sin embargo la revisión de los resultados de estos estudios, muestra información confusa, con ausencia de referentes 
teóricos claros y recurrentemente investigaciones que utilizan instrumentos para evaluar actitudes hacia la matemática, con diversas carencias tanto en los procesos de elaboración como en su validación (Ormerod \& Duckworth, 1975; Schibeci, 1984; Shrigley \& Koballa, 1992).

Entre las insuficiencias halladas destaca el abordaje de la propia variable de estudio. Las actitudes deben ser inferidas de las conductas manifiestas -imposible observarlas directamente, de ser así se parte desde elementos poco claros- con lo que conduce a establecer fundamentos teóricos inconsistentes $\mathrm{y}$, en consecuencia, un abordaje metodológico deficiente. Por otro lado, la mayoría de los instrumentos se han aplicado en poblaciones estudiantiles de los primeros años de bachillerato. Aunque existen referencias de aplicación en poblaciones universitarias, la tendencia ha sido circunscribir la indagación a un grupo limitado de carreras (Gal \& Ginsburg, 1994; Gómez-Chacón, 2002; Schuessler, 1993).

Teniendo en cuenta las limitantes señaladas, al conceptuar el constructo "Actitudes hacia la matemática", este trabajo inicia con un breve análisis teórico del término "actitudes", con base en sus componentes constitutivos: afectivos, cognoscitivos y conductuales, para derivar en la definición, en primer lugar de actitudes hacia la matemática y en segundo lugar, en la definición de sus categorías: actitudes positivas / negativas hacia la matemática, variables abordadas en este estudio.

En cuanto a la calidad de instrumentos para medir actitudes hacia la matemática, el presente estudio refiere el proceso de construcción y los procedimientos de validez y confiabilidad utilizados para validar psicométricamente el "Cuestionario de Actitudes hacia la matemática" -en lo adelante denominado CAM- diseñado en el año 2007, en el marco de una investigación sobre nuevos abordajes en la enseñanza-aprendizaje de la matemática en estudiantes universitarios.

Este trabajo conducido años más tarde, versa sobre la revalidación de las propiedades de este instrumento (CAM). Para ello, se aplicó nuevamente en el año 2013 a estudiantes de una institución universitaria de tecnología, en carreras de diseños curriculares con fuerte contenido de la asignatura de matemática, así como otras asignaturas cuyo sustrato es el conocimiento matemático. De esta manera, en este estudio se plantearon dos objetivos fundamentales: 
1. Revalidar los procedimientos estadísticos del "Cuestionario de Actitudes hacia la matemática" (CAM)

2. Analizar la consistencia de las propiedades psicométricas del instrumento.

\section{Actitudes... pensar, sentir, hacer}

¿Cómo deben ser definidas las actitudes? Se trata de una respuesta complicada a juzgar por la diversidad de definiciones del término, así como por la abundancia de posiciones teóricas que intentan explicar la cognición, emociones y conductas manifiestas como sus componentes (Ajzen \& Fishbein, 2005).

En la actualidad prevalecen los conceptos que definen las actitudes como tendencias evaluativas, bajo una concepción multidimensional, ajustada a las exigencias y flexibilidad teórica- metodológica de su definición operacional. Además, abarcan un espacio lo suficientemente extenso para permitir a los investigadores comprender los alcances y profundizar en su estudio como constructo psicológico (Eagly \& Chaiken, 1995, 2005; Fishbein \& Ajzen, 1975; Schwarz \& Bohner, 2001; Zanna \& Rempel, 1988).

Bajo estas premisas, en este trabajo se asumió como concepto de actitudes la planteada por Eagly y Chaiken (2005) y Zanna y Rempel (1988), quienes la definen como “tendencias psicológicas que se expresan valorando una determinada entidad con algún grado de predilección o desaprobación expresado a través de respuestas cognoscitivas, afectivas y conductuales" (p.11). Asumida desde esta perspectiva teórica, ¿qué implicaciones tiene para su estudio considerar las actitudes como sentencias de evaluación?

En primer lugar, los individuos pueden tener sentimientos ambivalentes hacia objetos concretos y sus actitudes implícitas y explícitas pueden diferir. Respecto de la ambivalencia, ésta puede surgir de varias fuentes y cuestiona la idea tradicional que las actitudes están ubicadas sobre un continuo bipolar (Eagly \& Chaiken, 1995; Fabrigar, MacDonald, \& Wegener, 2005). Por otro lado, esta perspectiva permite entender a las actitudes como tendencias positivas y negativas coexistentes, donde las respuestas de cada tendencia tienen diferentes grados de expresiones afectivas y cognoscitivas, así como diferentes efectos en el comportamiento. Todos estos componentes, llevan implícito el carácter de acción evaluativa, en tanto que las respuestas cognoscitivas, afectivas y conductuales no son más que su expresión externa.

En segundo lugar, un mismo individuo puede tener actitudes implícitas y explícitas 
hacia el mismo objeto actitudinal. En este sentido, las actitudes implícitas, pueden dirigir la respuesta, especialmente en presencia de comportamientos más espontáneos. Por el contrario, las actitudes explícitas, a las que el individuo tiene acceso deliberado, pueden ser activadas en una manera intencional, lo que requiere un esfuerzo cognoscitivo (Wilson, Lindsey, \& Schooler, 2000). Con relación a la expresión explícita e implícita de las actitudes, se maneja la hipótesis de que las actitudes subsisten en un continuo implícitoexplícito, dependiendo del grado en el que el individuo tiene acceso consciente a ellas, por tanto, las actitudes pueden resultar a veces ambiguas, ausentes o, por el contrario, claramente definidas (Wilson et al., 2000).

En tercer lugar, esta definición evaluativa, permite incluir la variabilidad de las actitudes a lo largo de una dimensión temporal, las sentencias actitudinales son formuladas nuevamente en cada ocasión que enfrenta un objeto de actitud, porque tales sentencias son influidas por el contexto específico en el que tienen lugar (Wilson \& Hodges, 1992). En cuarto y último lugar, reconoce su aspecto cognoscitivo, como creencias o representaciones mentales con un contenido específico de evaluación, que pueden ser construidas a partir de elementos posibles de presentarse en forma de afectos, ideas, creencias o comportamientos (Krosnick, Judd, \& Wittenbrink, 2005).

Así, Eagly y Chaiken (2005) argumentan que las actitudes pueden formarse a través de procesos cognoscitivos, afectivos y conductuales y expresadas a través de respuestas cognoscitivas, afectivas y conductuales. Estos componentes representan de la mejor manera posible, los tipos de respuesta que permiten a los investigadores el diagnóstico de actitudes. Agregan que, las personas elaboran actitudes con base en su conocimiento, afectos y respuestas conductuales a una entidad, asumiendo esos componentes como piezas interactivas más que separadas, es decir, las actitudes consisten en la evaluación general que proporciona la información derivada de esos elementos (Cacioppo, Petty \& Geen, 1989; Zanna \& Rempel, 1988). Finalmente, ponemos de relieve la importancia de tener claro el alcance teórico del constructo actitudes, toda vez que puede brindar un soporte teórico sólido para el análisis de respuestas actitudinales de estudiantes ante el aprendizaje matemático.

\section{Actitudes hacia la matemática}

Como se ha dicho, en este trabajo se partió de un marco teórico que concibe el 
estudio de las actitudes como sentencias evaluativas, con soportes suficientemente consolidados desde la perspectiva psicológica (Eagly \& Chaiken, 1995, 1998, 2005; Zanna \& Rempel, 1988). En consecuencia, la variable actitudes hacia la matemática se definió como "tendencias psicológicas evaluativas, inferidas a partir de ciertas respuestas cognoscitivas, afectivas y conductuales, que expresan en conjunto algún grado de aprobación o desaprobación hacia la matemática" (Farías, 2011, p. 57).

Bajo este marco referencial, se habla de dos categorías: actitudes positivas y actitudes negativas hacia la matemática, donde la primera se refiere a tendencias psicológicas evaluativas, inferidas a través de la expresión de creencias propicias, valoración emocional de agrado y conductas explícitas positivas, que en conjunto, manifiestan una apreciación favorable del individuo -que se va conformando a través de su desempeño- sobre sí mismo y de su relación con la matemática. Esa apreciación favorable propicia en el estudiante, que frente a dificultades explore nuevas vías de trabajo, que exhiba conductas relacionadas con la curiosidad, motivación, confianza e interés, elementos que en definitiva, influyen positivamente en el modo de enfocar las estrategias de aprendizaje. En el mismo tenor, la segunda categoría se establece como tendencias psicológicas evaluativas, inferidas a través de la expresión de creencias adversas, valoración emocional de desagrado y conductas explícitas negativas, que en conjunto, manifiestan una apreciación desfavorable, que va conformando el individuo sobre sí mismo y su relación con la matemática. Estas predisposiciones propician manifestaciones de inseguridad, decrecimiento del rendimiento académico, mínimo esfuerzo al realizar tareas, asunción de las dificultades como fracasos insuperables, renuencia a recibir ayuda, desconcierto, derrotismo, desesperanza, escasa participación en el salón de clases y exhibición de conductas disruptivas, ante situaciones de aprendizaje matemático.

\section{MÉTODO}

\section{Participantes}

Tanto en el proceso de construcción y validación del instrumento aplicado en 2007 como en su revalidación, año 2013, la participación de las personas fue voluntaria. En 2007 se seleccionaron intencionalmente estudiantes de las universidades Central de Venezuela (UCV), Simón Bolívar (USB), Católica Andrés Bello (UCAB) y Metropolitana (UNIMET). En tanto que la revalidación se realizó exclusivamente con estudiantes del Instituto 
Universitario de Tecnología Dr. Federico Rivero Palacio. Los siguientes criterios fueron compartidos en ambos procesos (validación / revalidación): alumnos inscritos en carreras cuyas mallas curriculares incluyeran materias de contenido matemático: matemática, cálculo, análisis, álgebra, probabilidades, estadística, matemática financiera /empresarial, entre otras. Género masculino y femenino, con edades correspondientes y cursantes de primero o segundo año de la carrera, equivalente a primero a cuarto semestre o entre el primero y octavo trimestre de la carrera según organización del plan de estudio.

\section{Instrumento}

En el diseño original del instrumento CAM, se establecieron a priori dos dimensiones: actitudes positivas y actitudes negativas hacia la matemática con sus respectivos reactivos, manteniendo abierta la posibilidad de una redefinición en función de los resultados obtenidos. Es importante destacar que en las reestructuraciones posteriores se mantuvieron las dimensiones mencionadas (Farías, 2011). El cuestionario se instrumentó a través del autorreporte, procedimiento que a partir de una serie de enunciados, estructurados en una escala, permite preguntar al estudiante qué siente, qué piensa o que está dispuesto a hacer respecto de la matemática. La escala utilizada fue de tres alternativas de respuesta ("nunca, a veces, siempre"). La selección de una escala de tres opciones de respuestas estuvo sustentada en que, en un continuo de tres elecciones es más fácil decidir exactamente dónde está la división conceptual entre oponer, adoptar la neutralidad o favorecer. Provee, además, mayor probabilidad de lograr consenso en el significado de las alternativas, lo que permite al participante identificar su propia conducta en términos conceptuales (lo hago o no; me gusta o no; estoy de acuerdo o no) y ubicarla en la opción más exacta. Finalmente, permite trabajar con los datos en su forma categórica, se pueden establecer proporciones de respuestas susceptibles de efectuarle cálculos de tendencia central, de dispersión y análisis de tablas de contingencia, mediante métodos no paramétricos convencionales (Krosnick, Judd, \& Wittenbrink, 2005).

Para cerrar, es necesario destacar dos aspectos importantes: primero, la medición de las actitudes de aceptación o rechazo hacia la matemática, -expresadas por las opiniones de la persona- estarían representando lo que el participante piensa, siente y hace con respecto a la asignatura. Segundo, al medir las actitudes, se está asumiendo que eventualmente el participante necesariamente actúa conforme a lo expresado, ya que pudiera ocultar sus 
verdaderas actitudes o, la presión social lo compele a creer lo que expresó, es decir, sus opiniones podría no reflejar forzosamente lo que realmente haría.

\section{Procedimiento}

El procedimiento se presentará en dos segmentos: el proceso de construcción del instrumento y el proceso de validación de la versión definitiva del CAM (2007). Lo realizado en el proceso de revalidación se mostrará en el apartado de los resultados.

\section{Proceso de construcción del CAM}

Previo al diseño del instrumento original (2007) se llevaron a cabo un conjunto de actividades preliminares que conducirían a su diseño definitivo:

\section{- Indagatoria a estudiantes y docentes acerca de su relación con la matemática}

Se realizó una exploración con estudiantes y docentes sobre factores actitudinales hacia la matemática con el objetivo de contar con proposiciones lo más ajustadas al objeto de estudio. Se partió del supuesto teórico de que las actitudes hacia la matemática están presentes en el proceso de aprendizaje. Sin embargo, por razones de orden metodológico, se realizó una primera búsqueda a través de entrevistas cortas semi-estructuradas, a efectos de confirmar su existencia o no.

Se entrevistaron veinte estudiantes de diferentes instituciones universitarias (Instituto Universitario de Tecnología "Dr. Federico Rivero Palacio, Universidad Metropolitana, Universidad Católica Andrés Bello, Universidad Central de Venezuela) cursantes de las carreras de física, matemática, biología, ingeniería de sistemas, ingeniería mecánica, tecnología de los materiales, administración, contaduría, comunicación social y letras. Se abarcaron tópicos como valoración académica y social de la asignatura, factores de éxito o fracaso en la materia, percepción como aprendices, actividades que realizan para aprender el contenido matemático.

Por otra parte, se interrogaron a ocho docentes -dos educadores matemáticos, tres matemáticos y tres físicos, profesores de matemática o física de las universidades Simón Bolívar, Católica Andrés Bello y Central de Venezuela, sobre sus roles en la enseñanza de la matemática (qué dicen, qué hacen, cómo se sienten en el aula impartiendo conocimiento), posibles fallas emocionales y cognoscitivas, atribuidas a los estudiantes al estudiar matemática y valoración académica-social de la asignatura. La información de los 
docentes fue utilizada para complementar la de los estudiantes.

Se analizó el material recolectado y las ideas seleccionadas fueron organizadas de acuerdo a su correspondencia con los indicadores en cada una de las categorías creadas (actitudes negativas / positivas hacia la matemática). Se procedió a la elaboración de un instrumento indagatorio, utilizando una escala si/no, conformado por ciento siete (107) proposiciones, que recogió las opiniones de los sujetos entrevistados y las propuestas de la autora y fue aplicado a un grupo de sesenta (60) estudiantes del Instituto Universitario de Tecnología Dr. Federico Rivero Palacio, cursantes del primer año en las carreras de Administración-Recursos Humanos (28), Ingeniería Mecánica (15) e Ingeniería de Materiales (17).

\section{- Evaluación de la comprensión de las proposiciones}

Conformado el instrumento, y a efectos de aumentar la comprensión de los estudiantes sobre los términos utilizados en cada una de las proposiciones, se diseñó una prueba para medir su comprensión del significado de los vocablos centrales de cada ítem, lo más ajustado al repertorio del estudiante. Se elaboró un instrumento con cincuenta y seis (56) palabras denominado “Cuestionario de exploración de reconocimiento verbal” (revisado por un licenciado en Letras y un doctor en Lingüística) utilizado para verificar la correspondencia entre lo que se deseaba medir y lo que realmente entendía el estudiante. Se aplicó el instrumento a sesenta (60) sujetos del Instituto Universitario de Tecnología Dr. Federico Rivero Palacio, cursantes del primer año en las carreras de AdministraciónMercadeo, Administración-Recursos Humanos, Ingeniería Mecánica e Ingeniería de Materiales.

\section{- Diseño de la versión preliminar del instrumento}

Con estos resultados se diseñó una versión preliminar del instrumento estructurada en tres partes: la primera, con veinte y cinco (25) ítems referidos a actitudes positivas; veinte y cinco (25) a actitudes negativas, para un total de cincuenta (50) ítems dispuestos aleatoriamente. La segunda, con cuatro (04) ítems para recoger información socioeconómica de los participantes y la tercera con cuatro (04) preguntas destinadas a obtener un registro histórico del desempeño del informante en matemática durante diferentes etapas académicas, en una escala de cinco opciones. Esta versión fue sometida a 
juicio de expertos y de acuerdo con sus evaluaciones se eliminaron catorce (14) ítems y se modificó la escala a tres opciones. (Farías, 2011).

\section{- Elaboración y aplicación de la primera versión del instrumento}

De acuerdo con las recomendaciones de los expertos, se realizó la primera versión del CAM, constituida por treinta y seis (36) proposiciones, manteniendo la indagación del estrato socioeconómico y del desempeño en la asignatura de matemática. Se llevó a cabo una prueba piloto del instrumento, para ello se le administró a un grupo de cuatrocientos (400) sujetos, estudiantes de las universidades Central de Venezuela, Simón Bolívar, Católica Andrés Bello y Metropolitana, cursantes de los primeros años de diversas carreras cuyas mallas curriculares incluyeran la asignatura de matemática o materias afines que la utilizan como soporte instrumental.

\section{Proceso de validación del CAM (2007)}

- Elaboración y aplicación de la versión definitiva del instrumento

A los datos obtenidos -producto de la prueba piloto ejecutada-, se le realizó un análisis factorial de componentes principales. Con base en los resultados arrojados se reestructuró el instrumento en su versión definitiva (Farías, 2011). Quedó constituido por veintiocho (28) ítems, manteniendo las otras partes sin modificaciones. Fue aplicada a un grupo de mil ciento setenta y nueve (1179) estudiantes, seleccionados conforme a los criterios ya mencionados, durante el primer semestre del 2007. La tabla 1 muestra la estructura del CAM en sus versiones:

Tabla 1. Estructura de las versiones del CAM.

\begin{tabular}{|c|c|c|c|c|}
\hline CAM & $\begin{array}{l}\text { Versión } \\
\text { Preliminar }\end{array}$ & $\begin{array}{l}\text { Primera } \\
\text { Versión }\end{array}$ & $\begin{array}{c}\text { Versión } \\
\text { Definitiva (2007) }\end{array}$ & $\begin{array}{c}\text { Versión } \\
\text { Revisada (2013) }\end{array}$ \\
\hline Número de Ítems & 50 & 36 & 28 & 27 \\
\hline & Actitudes +25 & Actitudes +17 & Actitudes +13 & Actitudes +13 \\
\hline & Actitudes - 25 & Actitudes - 19 & Actitudes - 15 & Actitudes - 14 \\
\hline Escala & 5 opciones & 3 opciones & 3 opciones & 5 opciones \\
\hline Participantes & & 400 & 1179 & 404 \\
\hline $\begin{array}{l}\text { Instituciones } \\
\text { Universitarias }\end{array}$ & $\begin{array}{c}\text { Expertos } \\
\text { Educación Matemática } \\
\text { Psicología del } \\
\text { Aprendizaje }\end{array}$ & $\begin{array}{c}\text { UCV, USB, } \\
\text { UCAB, UNIMET }\end{array}$ & $\begin{array}{c}\text { UCV, USB, } \\
\text { UCAB, UNIMET }\end{array}$ & IUT-RC \\
\hline
\end{tabular}


Continuando con el proceso de validación de la versión definitiva del CAM, se refiere enseguida cómo se hizo el análisis de validez y confiablidad estadística.

\section{- Análisis de Validez.}

No existen criterios únicos para establecer la validez estadística de un instrumento. En este caso la validez se estableció como sigue:

1. Construcción de enunciados. Como se señaló anteriormente, a partir de la indagatoria con estudiantes y docentes, se diseñó un instrumento, con una escala si / no, con el propósito de ratificar la existencia o no, de rasgos actitudinales en estudiantes universitarios. De las 107 proposiciones que conformaron la prueba, se seleccionaron 50, cuyo valor obtenido fue igual o mayor del $75 \%$ en la opción “si". Las restantes fueron eliminadas. La tabla 2 muestra solo las propuestas actitudinales elegidas:

Tabla 2. Proposiciones actitudinales positivas y negativas seleccionadas

\begin{tabular}{|c|c|c|c|c|c|c|}
\hline \multirow[t]{2}{*}{ No. } & \multirow{2}{*}{$\begin{array}{c}\text { Proposiciones } \\
\text { Actitudes hacia la matemática } \\
\mathbf{A}+/ \mathbf{A}-\end{array}$} & \multicolumn{2}{|c|}{ SI } & \multicolumn{2}{|c|}{ NO } & \multirow[t]{2}{*}{ Selección } \\
\hline & & f & $\%$ & $\mathbf{f}$ & $\%$ & \\
\hline 1 & $\begin{array}{l}\text { El profesor explica cosas fáciles y en los exámenes pregunta cosas difíciles. } \\
\text { A- }\end{array}$ & 45 & 75 & 15 & 25 & Elegido \\
\hline 2 & $\begin{array}{l}\text { Resolver problemas matemáticos estimula mi capacidad de razonamiento y } \\
\text { comprensión. } \mathbf{A}^{+}\end{array}$ & 48 & 80 & 12 & 20 & Elegido \\
\hline 3 & Cuando no entiendo matemática, me rindo. A- & 56 & 93 & 4 & 7 & Elegido \\
\hline 4 & Lo que aprendo en matemática tiene significado para mí. A+ & 55 & 92 & 5 & 8 & Elegido \\
\hline 5 & Temo equivocarme al resolver un problema matemático. A- & 57 & 95 & 3 & 5 & Elegido \\
\hline 6 & $\begin{array}{l}\text { En las clases de matemática el profesor motiva actitudes favorables hacia la } \\
\text { materia. } \mathbf{A}+\end{array}$ & 45 & 75 & 15 & 25 & Elegido \\
\hline 7 & $\begin{array}{l}\text { Me esfuerzo en utilizar diferentes estrategias de razonamiento al abordar una } \\
\text { actividad matemática. A+ }\end{array}$ & 49 & 82 & 11 & 18 & Elegido \\
\hline 8 & $\begin{array}{l}\text { El profesor permite que le diga con mis propias palabras lo que entendí sobre } \\
\text { el contenido dado en clase. A+ }\end{array}$ & 45 & 75 & 15 & 25 & Elegido \\
\hline 9 & $\begin{array}{l}\text { Aprendo mejor matemática cuando existe una comunicación afectiva con el } \\
\text { profesor. A+ }\end{array}$ & 45 & 75 & 15 & 25 & Elegido \\
\hline 10 & El profesor transmite un conjunto de reglas y fórmulas matemáticas. A- & 45 & 75 & 15 & 25 & Elegido \\
\hline 11 & En las clases de matemática siento confusión. A- & 56 & 93 & 4 & 7 & Elegido \\
\hline 12 & A pesar de mis esfuerzos salgo mal en matemática. A- & 55 & 92 & 5 & 8 & Elegido \\
\hline 13 & Estudiar matemática me motiva. A+ & 48 & 80 & 12 & 20 & Elegido \\
\hline 14 & El profesor relaciona lo dado en clase con nuevos contenidos. $\mathbf{A}+$ & 45 & 75 & 15 & 25 & Elegido \\
\hline 15 & Siento desagrado de sólo pensar que tengo que estudiar matemática. A- & 49 & 82 & 11 & 18 & Elegido \\
\hline 16 & El profesor se molesta cuando le digo que no entiendo. A- & 45 & 75 & 15 & 25 & Elegido \\
\hline 17 & El profesor establece relaciones entre el conocimiento matemático y mi & 45 & 75 & 15 & 25 & Elegido \\
\hline
\end{tabular}


realidad cotidiana. A+

18 Me gusta la matemática. A+

19 Estudio matemática únicamente para aprobar la materia. A-

$\begin{array}{lllll}50 & \mathbf{8 3} & 10 & 17 & \text { Elegido }\end{array}$

20 En las clases de matemática siento incapacidad. A-

21 Concibo la matemática como un juego que reta. A+

22 El profesor verifica cómo nos sentimos anímicamente durante la clase de matemática. A+

23 El profesor le da más importancia a la aplicación que a los conceptos matemáticos. A+

24 Las clases de matemática me interesan. A+

25 Siento que la matemática es útil cuando la aplico en mi vida diaria. A+

26 Para mí, la matemática es interesante. A+

27 El profesor se interesa más en enseñar el proceso, que en la solución del problema. A+

28 Me agrada experimentar varias vías para resolver problemas matemáticos. A+

29 Aplico lo que aprendí en matemática cuando realizo diseños tecnológicos e industriales. A+

30 Si apruebo un examen de matemática es porque el profesor se equivocó corrigiendo. A-

31 Estudio matemática por obligación. A-

32 Tengo confianza en mí mismo cuando enfrento problemas matemáticos. A+

33 Siento que fracaso en las clases de matemática. A-

34 Siento seguridad en las clases de matemática. A+

35 Por inseguridad no pienso con claridad lo que debo hacer ante un problema de matemática. A-

36 Me acelero y empiezo la tarea matemática con rapidez, sin detenerme a pensar que estoy haciendo. A-

37 Me aburro en clase de matemática $\mathbf{A}$ -

38 Controlo el temor que siento al enfrentarme a un problema matemático. A+

39 Siento que me falta tiempo para comprender el problema. A-

40 Me agradan las clases de matemática. A+

41 Siento inseguridad en las clases de matemática. A-

$\begin{array}{lllll}55 & 92 & 5 & 8 & \text { Elegido }\end{array}$

$\begin{array}{lllll}51 & 85 & 9 & 15 & \text { Elegido }\end{array}$

$\begin{array}{lllll}49 & 82 & 11 & 18 & \text { Elegido }\end{array}$

$\begin{array}{lllll}45 & 75 & 15 & 25 & \text { Elegido }\end{array}$

$\begin{array}{lllll}45 & 75 & 15 & 25 & \text { Elegido }\end{array}$

$\begin{array}{lllll}49 & 82 & 11 & 18 & \text { Elegido }\end{array}$

$\begin{array}{lllll}52 & 87 & 8 & 13 & \text { Elegido }\end{array}$

$\begin{array}{lllll}53 & 88 & 7 & 12 & \text { Elegido }\end{array}$

$\begin{array}{lllll}45 & 75 & 15 & 25 & \text { Elegido }\end{array}$

$\begin{array}{lllll}48 & 80 & 12 & 20 & \text { Elegido }\end{array}$

$\begin{array}{lllll}46 & 77 & 14 & 23 & \text { Elegido }\end{array}$

$\begin{array}{lllll}45 & 75 & 15 & 25 & \text { Elegido }\end{array}$

$\begin{array}{lllll}53 & 88 & 7 & 12 & \text { Elegido }\end{array}$

$\begin{array}{lllll}50 & 83 & 10 & 17 & \text { Elegido }\end{array}$

$\begin{array}{lllll}55 & 92 & 5 & 8 & \text { Elegido }\end{array}$

$\begin{array}{lllll}49 & 82 & 11 & 18 & \text { Elegido }\end{array}$

$\begin{array}{lllll}56 & 93 & 4 & 7 & \text { Elegido }\end{array}$

$\begin{array}{lllll}48 & \mathbf{8 0} & 12 & 20 & \text { Elegido }\end{array}$

$\begin{array}{lllll}51 & 85 & 9 & 15 & \text { Elegido }\end{array}$

$\begin{array}{lllll}50 & \mathbf{8 3} & 10 & 17 & \text { Elegido }\end{array}$

$\begin{array}{lllll}54 & 90 & 6 & 10 & \text { Elegido }\end{array}$

42 Me cuesta aplicar estrategias matemáticas para resolver problemas en mi vida diaria. A-

43 Me agrada estudiar matemática cuando cuento con el apoyo del profesor y de mis compañeros. A+

44 Siento contrariedad en las clases de matemática. A-

$\begin{array}{lllll}52 & 87 & 8 & 13 & \text { Elegido }\end{array}$

$\begin{array}{lllll}54 & 90 & 6 & 10 & \text { Elegido }\end{array}$

$\begin{array}{lllll}47 & \mathbf{7 8} & 13 & 22 & \text { Elegido }\end{array}$

$\begin{array}{lllll}45 & 75 & 15 & 25 & \text { Elegido }\end{array}$

45 Me da rabia cuando repito los mismos errores estudiando matemática. A-

$\begin{array}{lllll}50 & 83 & 10 & 17 \quad \text { Elegido }\end{array}$

46 Me desanima no lograr buenos resultados en matemática. A-

47 Me entristece saber que mis calificaciones en matemática son bajas. A-

$\begin{array}{lllll}51 & 85 & 9 & 15 & \text { Elegido }\end{array}$

$\begin{array}{lllll}54 & 90 & 6 & 10 & \text { Elegido }\end{array}$

48 Temo equivocarme cuando paso al pizarrón a resolver un problema $\begin{array}{llll}56 & 93 & 4 & 7\end{array}$

Elegido matemático. A-

49 El profesor me enseña diferentes estrategias para resolver problemas matemáticos. A+

$\begin{array}{lllll}53 & \mathbf{8 8} & 7 & 12 & \text { Elegido }\end{array}$

50 Disfruto los retos que me presenta resolver problemas matemáticos. A+

$\begin{array}{lllll}45 & 75 & 15 & 25 & \text { Elegido }\end{array}$

$\begin{array}{lllll}50 & \mathbf{8 3} & 10 & 17 & \text { Elegido }\end{array}$ 
2. Aplicación del “Cuestionario de exploración de reconocimiento verbal”. El instrumento estuvo conformado por 56 vocablos que fueron elegidos a partir de los sustantivos y verbos de cada proposición seleccionada. Para cada palabra se propusieron cuatro sinónimos, cuatro parónimos y cuatro antónimos, para un total de doce significados posibles. Se estimó que el estudiante, estaría en capacidad de seleccionar hasta cuatro posibles significados que, a su juicio, significarían lo mismo que la palabra evaluada (si escogía sinónimos obtenía 1 punto; parónimos 0 y antónimos -1).

Los resultados se evaluaron de forma porcentual, de acuerdo con la cantidad de veces que los informantes seleccionaron los significados adecuados de la lista, para cada una de las cincuenta y seis (56) palabras. Siguiendo ese razonamiento, se tuvo por bien reconocida una palabra cuyo promedio de aciertos estuviera entre el 50 y 100\%. Todos los promedios cuyos valores estuvieron por debajo del $50 \%$ corresponderían a palabras que no fueron bien reconocidas. La tabla 3 muestra los promedios de aciertos obtenidos para cada una de las palabras evaluadas:

Tabla 3. Porcentajes de aciertos de palabras que conforman el cuestionario "Exploración de Reconocimiento Verbal"

\begin{tabular}{|c|c|c|c|c|c|c|c|c|}
\hline \multicolumn{9}{|c|}{$\begin{array}{c}\begin{array}{c}\text { Porcentajes de aciertos } \\
\text { Palabras evaluadas }\end{array} \\
\end{array}$} \\
\hline 1. & Aburrimiento & $77 \%$ & 21. & Analizo & $79 \%$ & 40. & Contrariedad & $78 \%$ \\
\hline 3. & Planteo & $71 \%$ & 22. & Motiva & $88 \%$ & 41. & Fáciles & $58 \%$ \\
\hline 4. & Agrado & $61 \%$ & 23. & Resolví & $56 \%$ & 42. & Desagrado & $73 \%$ \\
\hline 5. & Frustración & $95 \%$ & 24. & Chequeo & $64 \%$ & 43. & Planifico & $45 \%$ \\
\hline 6. & Éxito & $72 \%$ & 25. & Explican & $70 \%$ & 44. & Práctica & $49 \%$ \\
\hline 7. & Interpretar & $74 \%$ & 26. & Incapaz & $70 \%$ & 45. & Obligación & $64 \%$ \\
\hline 8. & Compruebo & $71 \%$ & 27. & Abstracta & $77 \%$ & 46. & Irritable & $66 \%$ \\
\hline 9. & Represento & $54 \%$ & 28. & Difíciles & $60 \%$ & 47. & Establezco & $53 \%$ \\
\hline 10. & Disfruto & $71 \%$ & 29. & Información & $73 \%$ & 48. & Formación & $76 \%$ \\
\hline 11. & Necesaria & $90 \%$ & 30. & Interés & $67 \%$ & 49. & Concebir & $41 \%$ \\
\hline 12. & Identifico & $58 \%$ & 31. & Rabia & $74 \%$ & 50. & Reviso & $54 \%$ \\
\hline 13. & Dato & $71 \%$ & 32. & Capacidad & $63 \%$ & 51. & Verifico & $64 \%$ \\
\hline 14. & Profesión & $70 \%$ & 33. & Separar & $52 \%$ & 52. & Enfrentar & $67 \%$ \\
\hline 15. & Controlo & $86 \%$ & 34. & Deficiente & $69 \%$ & 53. & Confusión & $68 \%$ \\
\hline 16. & Inteligente & $54 \%$ & 35. & Aterradora & $73 \%$ & 54. & Incapacidad & $74 \%$ \\
\hline 17. & Fracaso & $69 \%$ & 36. & Entendimiento & $62 \%$ & 55. & Reta & $66 \%$ \\
\hline 18. & Sirvan & $66 \%$ & 37. & Angustiado & $53 \%$ & 56. & Recuerdo & $68 \%$ \\
\hline 19. & Entender & $66 \%$ & 38. & Teórica & $68 \%$ & & & \\
\hline 20. & Inseguridad & $59 \%$ & 39. & Seguridad & $52 \%$ & & & \\
\hline
\end{tabular}


Estos resultados confirmaron que las proposiciones seleccionadas para conformar el instrumento estaban formadas por palabras reconocidas por los estudiantes, por consiguiente expresaban la adecuación de los ítems con las variables a medir: actitudes positivas y negativas hacia la matemática.

3. Validación de contenido. Este proceso permitió depurar las versiones del instrumento y eliminar enunciados ambiguos o poco comprensibles. Con este procedimiento se pudo establecer que los ítems del instrumento eran representativos del dominio de contenido del atributo o cualidad que se pretendía medir (Landero \& González, 2006). A diferencia de otros tipos de validación, la de contenido no puede ser estimada cuantitativamente (coeficientes o índices) y el procedimiento comúnmente empleado es el de juicio de expertos (Ruiz, 2002). De esta manera, se entregó a cuatro jueces con experiencia en el campo de la educación matemática y psicología del aprendizaje, información sobre lo que pretendía el cuestionario, así como su versión preliminar. Cada juez evaluó la pertinencia de los ítems para valorar opiniones referidas a actitudes hacia la matemática en poblaciones de estudiantes universitarios y recomendaron modificar la redacción de algunos y la eliminación de otros. Como consecuencia de estas observaciones se obtuvo una primera versión constituida por treinta y seis (36) reactivos (17 actitudes positivas/19 actitudes negativas), que finalmente, se reestructuró obteniéndose la versión definitiva con veintiocho (28) ítems (13 actitudes positivas/15 actitudes negativas) (Farías, 2011).

4. Análisis Factorial. Para continuar con la determinación de la validez y confirmar las variables del instrumento, se aplicó un análisis factorial de componentes principales con rotación varimax, con el propósito de reducir la matriz de los datos a un conjunto menor de componentes y facilitar su interpretación. En principio, se llevó a cabo el cálculo de las pruebas de adecuación muestral de Kaiser-Meyer-Olkin (KMO) y de esfericidad de Bartlett cuyos valores (la primera 0,939 y la segunda $\mathrm{p}<0.001$ ) permitieron aplicar el análisis factorial. Se estimó el porcentaje de la varianza total explicada, para conocer el porcentaje acumulado específico, que explica una cantidad determinada de la varianza total entre los datos (Hair, Anderson, Tatham, \& Black, 2005). Se tomó en consideración para la extracción de factores el criterio de Kaiser (autovalores mayores que 1) y la identificación clara de dos dimensiones (actitudes positivas / actitudes negativas hacia la matemática). 
Así, se obtuvo un porcentaje de 39\% de la varianza válida para dos factores (primer factor $25 \%$; segundo factor 14\%), lo cual cumplió con los criterios de validez establecidos (Landero \& González, 2006). Los resultados que arrojó el análisis factorial efectuado en la versión definitiva del CAM se encuentran en la primera investigación ya publicada (Ver Farías, 2011).

\section{- Análisis de Confiabilidad}

La modalidad utilizada para la determinación de la confiabilidad de las puntuaciones de los ítems de este instrumento fue la de consistencia interna de los factores a través del coeficiente Alpha de Cronbach. Los valores obtenidos para cada factor fueron: factor $1(\alpha=0.90)$, factor $2(\alpha=0.86)$. Estos coeficientes se encuentran dentro del rango de los valores considerados confiables, lo que se traduce en un instrumento con una consistencia interna satisfactoria y una confiabilidad adecuada (Landero \& González, 2006). Hasta aquí, los aspectos más relevantes del diseño y validación del CAM en su versión definitiva del año 2007. A continuación, se reseñan las actividades y resultados obtenidos en el proceso de revalidación año 2013.

\section{RESULTADOS}

\section{Aplicación de la versión revisada del CAM (2013)}

Para la ratificación de las propiedades psicométricas del instrumento, se administró a cuatrocientos cuatro (404) individuos durante el primer semestre del 2013, con los criterios ya mencionados. En esta ocasión fueron estudiantes del Instituto Universitario de Tecnología Dr. Federico Rivero Palacio, cursantes del primer año o trayecto, de carreras o programas nacionales de formación cuyas mallas curriculares incluyeran la asignatura de matemática y materias afines. En esta versión se eliminó el ítem 22, "Siento que me falta tiempo para comprender el problema", por considerar que medía una conducta autorreguladora más que una opinión hacia la matemática; quedó conformado por veinte y siete (27) ítems (13 actitudes positivas/14 actitudes negativas). Otra modificación, fue la escala, se volvió a la utilizada en la versión preliminar (totalmente en desacuerdo $=1$; en desacuerdo $=2$; Indeciso $=3 ;$ de acuerdo $=4$ y totalmente de acuerdo $=5$ ). Esta decisión 
estuvo basada en que se mantienen las ventajas que se mencionaron al utilizar una escala de tres alternativas, además de que la escogencia de las opciones de respuesta no difieren de manera significativa por lo que se mantiene la consistencia de la medición de la variable y en consecuencia del instrumento (Ver Anexo No. 1).

\section{Evaluación de la validez y confiabilidad del instrumento}

Las respuestas obtenidas en la aplicación del instrumento fueron transcritas a una base de datos a la que se le aplicó varias pruebas estadísticas para determinar la validez y confiabilidad del instrumento diseñado, para esto se utilizó el Programa Estadístico para Ciencias Sociales (SPSS) versión 21.

\section{- Análisis de Validez}

La validez del instrumento se realizó a través del análisis factorial. No se consideró la validación de contenido, ya que teóricamente los ítems siguen respondiendo a las necesidades de las tablas de especificaciones del constructo.

Análisis Factorial. Se aplicó un análisis factorial de componentes principales con rotación varimax para establecer la validez de constructo, lo cual permitió corroborar la adecuación de los datos al modelo teórico propuesto. Se calcularon las pruebas de adecuación muestral de Kaiser-Meyer-Olkin (KMO) y de esfericidad de Bartlett. La medida de suficiencia de muestreo KMO es un índice que mide el grado de intercorrelaciones o dependencia entre los datos de la matriz. Su rango es de 0 a 1 , los valores cercanos a 1 son aceptables, por debajo de 0,50 son inaceptables (Hair et al., 2005). El resultado fue de 0,922, lo que permite sostener la existencia de intercorrelaciones entre las variables que conforman la matriz de varianzas y confirma la existencia de factores subyacentes. Por su parte, la prueba de contraste de esfericidad de Barlett, indica la probabilidad estadística de correlaciones entre los datos de la matriz. Valores mayores o iguales a 0,05 indican independencia entre los datos, valores menores a 0,05 reflejan dependencia entre los mismos (Landero \& González, 2006). El valor observado para la revalidación del instrumento es de $p<0.001$; este resultado aunado al de la prueba $K M O$ hacen pertinente el análisis factorial para la matriz de datos obtenida.

Se empleó el análisis factorial de componentes principales con rotación varimax, pues se supuso que las dimensiones del instrumento mantenían ciertos niveles de 
independencia entre ellas. Los resultados reflejaron un porcentaje total de la varianza explicada de $41 \%$ para dos factores, el primer factor asociado a la dimensión "Actitudes positivas hacia la matemática" explicó el 30\%, mientras que el segundo correspondiente a la dimensión "Actitudes negativas hacia la matemática" lo hizo con un $12 \%$. Como se pudo constatar, estos resultados fueron consistentes con los encontrados en la validación del instrumento en el año 2007, resultaron acordes con los criterios de validez previamente establecidos, permitieron ser interpretados psicológicamente y de acuerdo con Hair y colaboradores (2005) fueron satisfactorios y aceptables en ciencias humanas y sociales, por la complejidad de los objetos de estudio en estas áreas de investigación.

Tabla 4. Valores de KMO, Barlett y porcentaje de Varianza explicada CAM: Versión definitiva (2007) / Versión revisada (2013)

\begin{tabular}{ccc}
\hline Pruebas Estadísticas & $\begin{array}{c}\text { Versión Definitiva } \\
\text { CAM-2007 }\end{array}$ & $\begin{array}{c}\text { Versión Revisada } \\
\text { CAM-2013 }\end{array}$ \\
\hline KMO & 0,939 & 0,922 \\
Barlett & $p<0,001$ & $p<0,001$ \\
& I factor $25 \%$ & I factor 30\% \\
$\begin{array}{c}\text { Porcentaje de Varianza } \\
\text { Explicada }\end{array}$ & II factor 14\% & II factor 12\% \\
& Total 39\% & Total 41\% \\
\hline
\end{tabular}

Finalmente, la tabla 5 muestra la matriz de correlaciones entre los factores y los ítems del instrumento (coeficientes de estructura) de la versión definitiva CAM-2007 (validación) y la versión revisada CAM-2013 (revalidación).

En este trabajo, se mantienen los resultados observados en el 2007 (Farías, 2011) y permiten corroborar el modelo teórico para la evaluación de las actitudes hacia las matemáticas. Consecuentes con el plan original se asumió la propuesta de Hair y colaboradores (2005) quienes fijan coeficientes de 0,35 como significativos para tamaños muestrales de 250 sujetos. En nuestro caso, tanto en las pruebas de validación como en la de revalidación, el número de participantes estuvo por encima de los trescientos cincuenta (350) sujetos, por tanto el criterio mínimo de coeficiente de estructura o carga factorial fue estimado en un valor de 0,350 , el cual fue definitorio para organizar la versión definitiva del CAM, puesto que se mantuvieron sólo los reactivos que lograron mayor peso y 
representatividad.

Tabla 5. Matriz de componentes rotados: Versión definitiva CAM (2007) / revalidación CAM (2013)

\begin{tabular}{|c|c|c|c|}
\hline \multirow{2}{*}{$\begin{array}{c}\text { FACTORES } \\
\text { Cuestionario de Actitudes hacia la matemática (CAM) }\end{array}$} & \multicolumn{2}{|c|}{ Correlaciones } & \multirow{2}{*}{$\begin{array}{l}\text { No. } \\
\text { ítems }\end{array}$} \\
\hline & $\begin{array}{l}\text { Versión } \\
\text { Definitiva } \\
\text { (28 ítems) }\end{array}$ & $\begin{array}{c}\text { Versión } \\
\text { Revalidada } \\
\text { (27 ítems) }\end{array}$ & \\
\hline \multicolumn{4}{|l|}{$\begin{array}{c}\text { PRIMER FACTOR } \\
\text { Actitudes positivas hacia la matemática }\end{array}$} \\
\hline Me gusta la matemática & 0,779 & 0,775 & 7 \\
\hline Me agradan las clases de matemática & 0,771 & 0,783 & 23 \\
\hline Estudiar matemática me motiva & 0,764 & 0,728 & 6 \\
\hline Las clases de matemática me interesan & 0,762 & 0,795 & 11 \\
\hline Para mí, la matemática es interesante & 0,742 & 0,852 & 13 \\
\hline Lo que aprendo en matemática es significativo para mí & 0,660 & 0,550 & 2 \\
\hline Estudio matemática por obligación & $-0,650$ & $-0,665$ & 15 \\
\hline Disfruto los retos que me presenta resolver problemas matemáticos & 0,613 & 0,620 & 28 \\
\hline Estudio matemática únicamente para aprobar la materia & $-0,590$ & $-0,660$ & 8 \\
\hline Concibo la matemática como un juego que reta & $\mathbf{0 , 5 8 5}$ & $\mathbf{0 , 3 4 3}$ & 10 \\
\hline $\begin{array}{l}\text { Me agrada plantearme varias vías para resolver problemas } \\
\text { matemáticos }\end{array}$ & 0,533 & 0,436 & 14 \\
\hline Me aburro en clase de matemática & $-0,528$ & $-0,519$ & 20 \\
\hline Siento que la matemática es útil cuando la aplico en mi vida diaria & 0,493 & 0,554 & 12 \\
\hline \multicolumn{4}{|l|}{$\begin{array}{c}\text { SEGUNDO FACTOR } \\
\text { Actitudes negativas hacia la matemática }\end{array}$} \\
\hline Me siento inseguro en las clases de matemática & 0,674 & 0,583 & 24 \\
\hline Por inseguridad me equivoco al resolver tareas matemáticas & 0,662 & 0,684 & 19 \\
\hline A pesar de que estudio salgo mal en matemática & 0,633 & 0,628 & 5 \\
\hline $\begin{array}{l}\text { Siento que fracaso al equivocarme cuando resuelvo tareas } \\
\text { matemáticas }\end{array}$ & 0,632 & 0,646 & 17 \\
\hline En las clases de matemática me siento incapaz & 0,619 & 0,605 & 9 \\
\hline Me siento contrariado en las clases de matemática & 0,589 & 0,614 & 25 \\
\hline Siento que me falta tiempo para comprender el problema & 0,574 & Eliminado & 22 \\
\hline En las clases de matemática me siento confundido & 0,545 & 0,621 & 4 \\
\hline Temo equivocarme al resolver un problema matemático & 0,536 & 0,581 & 3 \\
\hline Confío en mis capacidades cuando resuelvo tareas matemáticas & $-0,526$ & $-0,383$ & 16 \\
\hline Cuando no entiendo matemática, me rindo & 0,491 & 0,457 & 1 \\
\hline $\begin{array}{l}\text { Temo equivocarme cuando paso al pizarrón a resolver un problema } \\
\text { matemático }\end{array}$ & 0,487 & 0,635 & 27 \\
\hline $\begin{array}{l}\text { Controlo el temor que siento al enfrentarme a un problema } \\
\text { matemático }\end{array}$ & $-0,403$ & $-0,404$ & 21 \\
\hline Me siento seguro en las clases de matemática & $-0,398$ & 0,459 F1 & 18 \\
\hline Me entristece saber que mis calificaciones en matemática son bajas & 0,390 & 0,396 & 26 \\
\hline
\end{tabular}

Al igual que en el instrumento validado (CAM-2007) se obtuvo la misma estructura factorial (dos factores). Hubo dos ítems que marcaron diferencias, el reactivo "Me siento seguro en las clases de matemática", en el cuestionario original obtuvo un coeficiente de estructura negativo en el factor 2 (Actitudes negativas hacia la matemática), mientras que 
en la revalidación alcanzó un coeficiente positivo en el factor 1 (Actitudes positivas hacia la matemática). El ítem “Concibo la matemática como un juego que reta”, en la revalidación logró un coeficiente de estructura $(0,343)$ por debajo del criterio establecido $(0,350)$, en tanto que en la matriz de la versión definitiva consiguió un valor de 0,585. En el primer caso, el ítem sigue evaluando la misma actitud hacia la matemática; en el segundo caso, el ítem cargó en el mismo factor y reactivos con cargas por encima de 0,3 se consideran aceptables (Landero \& González, 2006). En términos generales, el comportamiento de los ítems se ha mantenido, pueden considerarse las pequeñas variaciones observadas como producto del muestreo, por lo que la consistencia psicométrica de la prueba original no fue afectada.

\section{- Análisis de confiabilidad}

El análisis de confiabilidad del instrumento se realizó a través del cálculo del coeficiente Alpha de Cronbach. Este procedimiento permitió determinar el grado en que los ítem están correlacionados entre sí, su valor oscila entre 0 y 1 (Ruiz, 2002). Cabe decir que existen diferentes propuestas acerca de cuál es el valor adecuado para indicar un coeficiente de confiabilidad en las puntuaciones de los reactivos de un instrumento, sin embargo, se observa una firme tendencia a considerar estas puntuaciones confiables si sus valores se ubican entre 0,70 a 1,00 (DeVellis, 2003; Kerlinger \& Lee, 2002; Landero \& González, 2006; Nunnally \& Berstein, 1995). Los valores obtenidos en el proceso de revalidación fueron los siguientes:

Factor 1: $\alpha=0,70 /$ Factor 2: $\alpha=0,79$

Landero y González (2006) sugieren como valores "muy altos" los ubicados entre 0,81 a 1,00 y "altos" los que se encuentran entre 0,61 a 0,80. Asumiendo estos criterios, los valores obtenidos presentaron consistencias "altas". Tanto en la validación como en la revalidación, los coeficientes obtenidos se encuentran dentro del rango de los valores considerados confiables, lo que se traduce en un instrumento con una consistencia interna satisfactoria y una confiabilidad adecuada. Es necesario destacar que, como lo muestra la tabla 6, los coeficientes en la revalidación fueron menores, esto se debió a que participaron menos individuos. Sin embargo, estos alfas permiten sostener que la prueba es aceptable y que la confiabilidad del instrumento no se redujo. 
Tabla 6. Coeficientes de Confiabilidad

\begin{tabular}{ccc}
\hline Alpha de Cronbach & $\begin{array}{c}\text { Versión Definitiva } \\
(\mathbf{2 8} \text { ítems/1179) }\end{array}$ & $\begin{array}{c}\text { Versión Revisada } \\
(\mathbf{2 7 / 4 0 4 )}\end{array}$ \\
\hline I factor & 0,90 & 0,86 \\
II factor & 0,70 & 0,79 \\
\hline
\end{tabular}

\section{DISCUSIÓN}

Es importante apuntar, que no existen criterios establecidos que indiquen procesos de validación aceptables o inaceptables en un instrumento. No obstante, el CAM alcanza valores similares a los obtenidos en algunos trabajos sobre la confirmación de propiedades estadísticas de instrumentos psicométricos (Hair et al., 2005; Ruiz, 2002). El clásico cuestionario para medir actitudes hacia la matemática en estudiantes, elaborado por Fennema y Sherman (1986) ha sido cuestionado en su validez y confiabilidad por investigadores como Suinn y Edwards (1982), sin embargo, este instrumento ha sido aplicado en múltiples ocasiones, se sigue usando en versiones actualizadas y su índice de confiabilidad de 0,87 es una referencia (Gómez, 1999). El CAM obtuvo en cada uno de sus factores - tanto en la validación como en la revalidación- valores superiores y cercanos a este índice.

La Escala de Actitudes hacia la Estadística y la Matemática de Auzmendi (1992), evalúa indistintamente actitudes hacia la estadística y hacia la matemática. Su estructura factorial arrojó cinco dimensiones, siendo 0,89 el mayor coeficiente y el menor de 0,66. Como se expuso, los factores del CAM obtuvieron valores que están dentro de los rangos logrados en este cuestionario.

Por último, se destaca uno de los trabajos realizados por investigadores del área de Didáctica de la Matemática en España, quienes en el marco de un estudio descriptivo diseñaron un cuestionario para evaluar actitudes de estudiantes hacia la matemática. El análisis de la validez y confiabilidad de este instrumento, arroja una consistencia interna elevada, muy parecida a la obtenida en el trabajo de revalidación del CAM (Hernández, Palarea, \& Socas, 2001; Hernández \& Socas, 1999).

Habiendo destacado la correspondencia entre los resultados obtenidos en la validación/revalidación del CAM con algunos instrumentos considerados como referencia 
imprescindible en la evaluación de la consistencia de pruebas para medir actitudes hacia la matemática, podría decirse que los resultados obtenidos en la revalidación psicométrica del "Cuestionario de actitudes hacia la matemática (CAM)" -tomando como punto de partida su validación en el año 2007-, confirman sus propiedades psicométricas y avalan su utilización con un grado satisfactorio de validez y confiabilidad, por las siguientes razones:

- Al corroborar la validez del CAM se obtuvieron dos factores, en los que la distribución de sus ítems resultó en íntima correspondencia con el marco teórico propuesto para el abordaje de las actitudes hacia la matemática, y se mantuvo la estructura factorial lograda en la validación llevada a cabo en el 2007.

- Sobre la base del Análisis Factorial se corroboró que el instrumento tal como ha sido estructurado posee validez de constructo. Se evidencia correspondencia entre el modelo teórico y las evidencias empíricas, es decir, se observa un ajuste en el modelo a la luz de los datos empíricos.

- En el año 2007 respondió a la propuesta teórica utilizada, en este trabajo de revalidación, la matriz de correlaciones observadas en la revalidación, permite sostener la propuesta teórica para las actitudes hacia la matemática.

- Con respecto a los coeficientes de estructura de los reactivos que conforman el instrumento, éstos superan el valor límite empleado en este estudio $(0,350)$. De igual manera, si tomamos como referencia el valor del coeficiente de estructura de 0,5 establecida para la Escala de Actitudes hacia la Matemática diseñada por Fennema y Sherman (1986), el 74\% de los ítems obtuvieron valores por encima de este coeficiente.

- Los coeficientes obtenidos en el análisis de confiabilidad para cada uno de los factores refieren una alta consistencia interna (validación/revalidación), también sugieren que cada uno de los factores explora constructos distintos, de manera tal que permite examinar con independencia entre una actitud positiva y una negativa hacia la matemática.

Finalmente, las propiedades psicométricas del Cuestionario de "Actitudes hacia la Matemática" sugieren a docentes e investigadores, que contar con un instrumento válido para medir actitudes hacia la matemática, proporciona información relevante que pudiera tener implicaciones pedagógicas importantes, en el diseño y ajustes de estrategias educativas tendentes a aumentar los logros académicos en matemática. 


\section{REFERENCIAS}

Ajzen, I., \& Fishbein, M. (2005). The influence of Attitudes on Behavior. In Albarracin, D. Johnson, B. \& Zanna M. (Eds.). The Handbook of Attitudes. New Jersey: Erlbaum.

Cacioppo, J., Petty, R., \& Geen, M. (1989). Attitude structure and function: From the tripartite to the homeostasis model of attitude. In A. Pratkanis, S., Breckler \& A. Greenwald (Eds.) Attitude structure and function. Hillsdale, NJ: Lawrence Erlbaum Associates.

DeVellis, R. (2003). Scale development. Theory and applications (segunda edición). Thousand Oaks, Sage Publications.

Eagly, A., \& Chaiken, S. (1995). Attitude strength, attitude structure, and resistance to change. In R. Petty \& J. Kronick (Eds.) Attitude strength: Antecedents and consequences. Mahwah, NJ: Lawrence Erlbaum Associates.

Eagly, A., \& Chaiken, S. (1998). Attitude structure and function. In D. Gilbert, S. Fiske \& G. Lindsey (Eds.) Handbook of social psychology. Boston: McGraw- Hill.

Eagly, A., \& Chaiken, S. (2005). Attitude Research in the 21st Century: The current state of knowledge. In Albarracin, D., Johnson, B. \& Zanna, M. (Eds.). The Handbook of Attitudes. New Jersey: Erlbaum.

Fabrigar, L., MacDonald, T., \& Wegener, D. (2005). The structure of attitudes. In Albarracin, D. Johnson, B. \& Zanna, M. (Eds.). The Handbook of Attitudes. New Jersey: Erlbaum.

Farías, M. (2011). Actitudes y Autorregulación en el aprendizaje de la matemática. Nuevos abordajes en la enseñanza-aprendizaje de la matemática en estudiantes universitarios. Editorial Académica Española: Alemania.

Fennema, E., \& Sherman, J. (1986). Fennema-Sherman Mathematics Attitudes Scale. Instrument Designed to Measure Attitudes towards the Learning of Mathematics by females and Males Wisconsin Center for Education Research School of Education. University of Wisconsin- Madison. Catalog of Selected Documents In Psychology, 6(1), 31-45.

Fishbein, M., \& Ajzen, I. (1975). Belief, attitude, intention, and behavior: An introduction to theory and research. Reading, MA: Addison- Wesley.

Gal, I., \& Ginsburg, L. (1994). The role of beliefs and attitudes in learning statistics: Towards an assessment framework. Journal of Statistics Education, 2(2), 37-51.

Gómez, I. (1999). Calculadoras gráficas y pre cálculo. Las actitudes de los estudiantes. Disponible en http://www.ort.edu.uy/REDOC/7-actitu.htm. Consulta: 2012, Octubre 15.

Gómez-Chacón, I. (2002). Afecto y aprendizaje matemático: causas y consecuencias de la interacción emocional. En J. Carrillo (ed.) Reflexiones sobre el pasado, presente y futuro de las matemáticas. Universidad de Huelva.

Hair, J., Anderson, E., Tatham, R., \& Black, W. (2005). Análisis Multivariante. Quinta Edición. Prentice Hall Iberia: Madrid.

Hernández, J., \& Socas, M. (1999). Las actitudes de los alumnos hacia la matemática. El papel de los materiales didácticos. En Socas, M., Camacho, M. y Morales, A. Formación del profesorado e 
investigación en educación matemática I. Departamento de análisis matemático. Universidad de La Laguna.

Hernández, J., Palarea, M., \& Socas, M. (2001). Análisis de las concepciones, creencias y actitudes hacia la matemática de los alumnos que comienzan a diplomatura de maestro. El papel de los materiales didácticos. En Socas, M., Camacho, M. y Morales, A. Formación del profesorado e investigación en educación matemática I. Departamento de análisis matemático. Universidad de La Laguna.

Kerlinger, F., \& Lee, H. (2002). Investigación del comportamiento. Métodos de investigación en ciencias sociales. Cuarta edición. México: McGraw Hill.

Krosnick, J., Judd, Ch., \& Wittenbrink, B. (2005). The measurement of attitudes. In Albarracin, D. Johnson, B. \& Zanna, M. (Eds.). The Handbook of Attitudes. New Jersey: Erlbaum.

Landero, R., \& González, M. (2006). Estadística con SPSS y metodología de la investigación. México: Trillas.

Morales, F. (1998). Actitudes de los escolares hacia la computadora y los medios para el aprendizaje. Tecnología y Comunicación Educativa, Junio-Diciembre. (28): 51-65.

Nunnally, J., \& Berstein, I. (1995). Teoría Psicométrica. México: Trillas.

Ormerod, M., \& Duckworth, D. (1975). Pupils attitudes to science: a review of research. Windsor: NFER Publishing.

Ruiz Bolívar, C. (2002). Instrumentos de Investigación Educativa. Procedimientos para su diseño y validación. Venezuela: Cideg.

Schibeci, R. (1984). Attitudes to science: an update. Studies in Science Education, 11, 26-59.

Schuessler, K. (1993). Scaling social life feelings: Persistent issues. In D. Krebs \& P. Schmidt (Eds.), New Directions in Attitude Measurement (21-40). Alemania: Walter De Gruyter.

Schwarz, K., \& Bohner, J. (2001). The construction of attitudes. In A. Tesser \& N. Schwarz (Eds.), Blackwell handbook of social psychology: Intraindividual processes. Oxford, UK: Blackwell.

Shrigley, R., \& Koballa J. (1992). A decade of attitude research base don Hovlands learning model. Science Education, 76(1), 17-42.

Suinn, R., \& Edwards, R. (1982). The measurement of mathematics anxiety: The mathematics anxiety rating scale for adolescent-mars-a. Journal of Clinical Psychology, 38(3), 576-580.

Wilson, T., Lindsey, S., \& Schooler, J. (2000). A model of dual attitudes. Psychological Review, 107(1), 101126.

Zanna, M., \& Rempel, J. (1988). Attitudes: a new look at an old concept. In D. Bar- Tal \& A. Kruglanski (Eds.). The social psychology of knowledge. Cambridge, UK: Cambridge University Press. 


\section{APÉNDICE}

\section{Anexo No. 1}

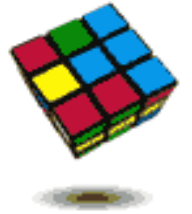

CAM

iversitaria

\section{Cuestionario de Actitudes hacia la Matemática \\ Farías, M. $(2007,2013)$}

Fecha

$\mathrm{PNF} /$ Carrera

Año/Semestre/Trimestre/Trayecto Edad Gen: M $\mathrm{F}$

El CAM, tiene como objetivo conocer apreciaciones hacia la matemática. Para ello, se presentan una serie de proposiciones sobre las actitudes hacia el aprendizaje matemático que con mayor frecuencia reportan estudiantes universitarios. Requerimos de tu participación al proporcionar la información solicitada con la mayor sinceridad. Por la naturaleza de los aspectos investigados no existen respuestas correctas o incorrectas.

Por favor lee con atención las proposiciones y selecciona para cada una la alternativa que mejor se ajuste a tu opinión. Es importante contestarlas todas y escoger sólo la opción que corresponda con tu apreciación, marcando con una $\underline{\mathbf{X}}$ en la columna correspondiente.

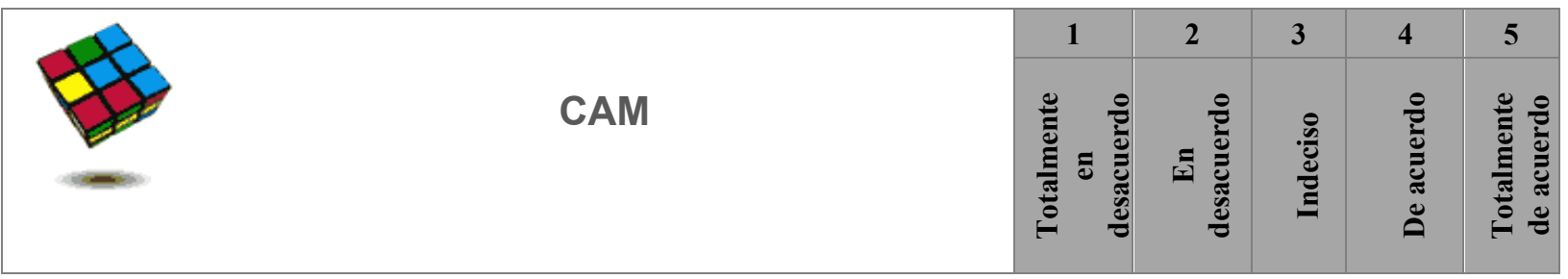

1. Cuando no entiendo matemática, me rindo

2. Lo que aprendo en matemática es significativo para mí

3. Temo equivocarme al resolver un problema matemático

4. En las clases de matemática me siento confundido

5. A pesar de que estudio salgo mal en matemática

6. Estudiar matemática me motiva

7. Me gusta la matemática

8. Estudio matemática únicamente para aprobar la materia

9. En las clases de matemática me siento incapaz

10. Concibo la matemática como un juego que reta 


\begin{tabular}{|c|c|c|c|c|c|c|}
\hline & & 1 & 2 & 3 & 4 & 5 \\
\hline$\Rightarrow$ & CAM & 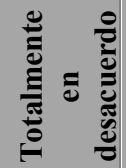 & 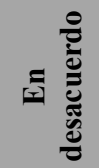 & 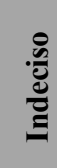 & 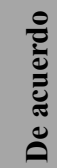 & 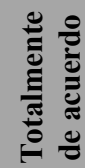 \\
\hline
\end{tabular}

11. Me interesan las clases de matemática

12. Siento que la matemática es útil cuando la aplico en mi vida diaria

13. Para mí, la matemática es interesante

14. Por inseguridad me equivoco al resolver tareas matemáticas

15. Estudio matemática por obligación

16. Confío en mis capacidades cuando resuelvo tareas matemáticas

17. Siento que fracaso al equivocarme cuando resuelvo tareas matemáticas

18. Me siento seguro en las clases de matemática

19. Me agradan las clases de matemática

20. Me aburro en clase de matemática

21. Controlo el temor que siento al resolver un problema matemático

22. Me agrada plantearme varias vías para resolver problemas matemáticos

23. Me siento inseguro en las clases de matemática

24. Disfruto los retos que me presenta resolver tareas matemáticas

25. Me entristece salir reprobado en matemática

26. Temo equivocarme cuando paso al pizarrón a resolver un problema matemático

27. Me siento contrariado en las clases de matemática 